\title{
Affective Advice Giving Dialogs
}

\author{
Addolorata Cavalluzzi, Valeria Carofiglio and Fiorella de Rosis \\ Intelligent Interfaces, Department of Informatics, University of Bari \\ \{cavalluzzi, carofiglio, derosis\}@di.uniba.it
}

\begin{abstract}
In affective dialog simulation, recognition and interpretation of the affective state of the user should be integrated with display of empathy by the system and with dialog planning and execution. Cognitive models dealing with the inherent uncertainty of this interpretation are the method we propose to adopt. We describe how we integrated these models in an information-state approach to dialog modeling by illustrating, in particular, their application to a decision support system which is tailored to the 'state of change' of the user.
\end{abstract}

\section{Introduction}

In computer-based advice-giving dialogs, the system aims at convincing the user to follow a given plan, by providing the information which is needed to increase the chance of success of its persuasive action. This chance of success increases if the system establishes an empathic relationship with the user: even in domains which might appear as neutral, empathy contributes to make advice more acceptable. Recognizing the user attitude and adapting the advice accordingly is more crucial when this concerns a course of action that, for some reason, the user may find difficult to follow: typically, cease smoking or change healthy eating habits. In this case, the amount and type of information provided has to be calibrated to the attitude of the user towards behaviour changing: this attitude includes her beliefs of what a 'correct' behaviour is, her intention to change behaviour if incorrect, her knowledge of a plan of how this goal may be achieved. Prochaska and colleagues (1992) proposed a theoretical model of behaviour change and a method to assess the state of a subject in this change and to find the 'best' advice-giving procedure in every stage. Their model became a reference for a large number of public health services all around the world.

Adoption of an advice-giving policy which is tailored to the attitude of the user requires, first of all, recognizing this attitude and, secondly, selecting the strategy to adopt from a library of known and experimentally evaluated alternatives. As this process occurs in a situation of uncertainty, both tasks have to be seen as dynamic processes, in which the system proceeds by a trial-and-error method. Dialogs are much more suited to this task than monologs, as they enable the system to monitor the situation, revise its hypothesis and re-adapt dynamically its strategy if needed.

A dialog model of this kind needs a sophisticated component of interpretation of the user moves. Not only beliefs, intentions and plans have to be inferred from analysis of these moves, but also the (stable or transient) 'affective' components of the user mind: her personality, mood and emotional state. On the other hand, selecting the appropri- 
ate advice-giving procedure alone is not a guarantee of success; the system has to show, at the same time, that it is not a 'purely rational' agent but shares part of the emotions of the user, in order to establish a relationship of trust and therefore to increase its chance of success. The system knows, finally, that what it will say will influence the user's rational and emotional attitude, that is her beliefs, intentions and plans; it may exploit this knowledge to achieve its goal of persuading the user to follow the appropriate behaviour. This exploitation is not unfair, because it is done 'for the interlocutor's sake'.

An advice-giving dialog system of this kind needs a sophisticated model of both the system and the user, which extends the well known BDI approach with a non-rational component (BDI\&E). It needs a dialog simulation method which adapts dynamically goals and plans to these two models and to a forecast of the possible effects of a candidate move on the user's affective and rational state. In this paper, we describe how we simulated such a dialog system by extending the 'information-state' model (Traum and Larsson, 2003); an example in the healthy eating domain will illustrate the method.

\section{Two Example Dialogs}

Let us start from the following excerpts of dialog:

Dialog1:

S3: I may try to help you, but cannot take a decision in your place.

U3: I would like to do something but it's so difficult!

S4: I know what you mean: diet is difficult to follow, particularly if you eat out of home because of work. But the effects may be very positive!

U4: Are you sure?

S5: Of course! Being in good shape can have beneficial effects on your appearance and health! I'm sure you know this, do you?

Dialog 2:

as in Dialog 1

U4: I know! I definitely intend to change my eating habits.

S5: Good! Why, for instance, don't you try to avoid daily lunches in a bar or high-fat sandwiches?

U5: Everyday you mean? I may try, but I must find an alternative.

S6: You may bring something from home: some fresh fruits or vegetables, for instances. And have a cup of tea or a fruit juice.

In these examples, the system interleaves suggestions with sentences aimed at assessing the user reaction and inferring her attitude. At move $\mathrm{U} 4$, in dialog 1 the user seems to show a 'doubtful' attitude about her problem behaviour and the system (in S5) tries to encourage evaluation of the advantages of changing it; in the second one, her answer is positive and suggests that she probably considers her behaviour as incorrect: the system that provides an initial suggestion of what to do. 


\section{State Of Change Model}

This model (Prochaska et al, 1992) describes how a subject changes of position from a 'wrong' behaviour in some domain to adoption of a 'correct' one. Six main stages are identified in this process of change (see Table 1).

Table 1: Signs and Actions in the SoC Model (from Prochaska et al, 1992)

\begin{tabular}{|c|c|c|}
\hline State of Change & Signs & Actions \\
\hline Pre-contemplation & $\begin{array}{l}\text { Does not want to change } \\
\text { behaviour, now or later } \\
\text { Believes that behaviour is } \\
\text { acceptable }\end{array}$ & $\begin{array}{l}\text { Validate lack of readiness } \\
\text { Clarify: decision is theirs } \\
\text { Encourage re-evaluation of current behaviour } \\
\text { Encourage self-exploration } \\
\text { Explain and personalize the risk }\end{array}$ \\
\hline Contemplation & $\begin{array}{l}\text { Doubts about behaviour } \\
\text { acceptable. } \\
\text { Thinks about why one follows } \\
\text { the bad habit, what its payoff is } \\
\text { Considers seriously the oppor- } \\
\text { tunity of changing behaviour } \\
\text { Does not want to commit to } \\
\text { change it soon }\end{array}$ & $\begin{array}{l}\text { Validate lack of readiness } \\
\text { Clarify: decision is theirs } \\
\text { Encourage evaluation of pros and cons of behaviour change } \\
\text { (bring both the rational mind and the emotions into play to } \\
\text { move you to a commitment to change) } \\
\text { Identify and promote new, positive outcome expectations }\end{array}$ \\
\hline Preparation & $\begin{array}{l}\text { Believes that behaviour should } \\
\text { be changed } \\
\text { Intends to change behaviour } \\
\text { soon }\end{array}$ & $\begin{array}{l}\text { Assist in problem solving } \\
\text { Provide social support } \\
\text { Verify that patients has underlying skills for behaviour } \\
\text { change } \\
\text { Provide counselling } \\
\text { Encourage initial steps: remove temptations, plan how } \\
\text { action will be taken, arrange support and understanding by } \\
\text { your neighborhoods } \\
\text { Suggest a follow-up plan }\end{array}$ \\
\hline Action & $\begin{array}{l}\text { Is already following a plan to } \\
\text { change behaviour (from some } \\
\text { months) }\end{array}$ & $\begin{array}{l}\text { Follow closely in order to sustain change of behaviour } \\
\text { Bolster self-efficacy for dealing with obstacles } \\
\text { Combat feelings of loss and reiterate long-term benefits }\end{array}$ \\
\hline Maintenance & $\begin{array}{l}\text { Is maintaining change from } \\
\text { more than } 6 \text { months }\end{array}$ & $\begin{array}{l}\text { Plan for follow-up support } \\
\text { Reinforce internal rewards } \\
\text { Discuss coping with relapse: learning the difference be- } \\
\text { tween lapse and a total relapse; be prepared to recognize a } \\
\text { lapse and take immediate action }\end{array}$ \\
\hline Relapse & $\begin{array}{l}\text { Went back to incorrect behav- } \\
\text { iour }\end{array}$ & $\begin{array}{l}\text { Reassess motivation and barriers } \\
\text { Assess state of change } \\
\text { Plan stronger coping strategies }\end{array}$ \\
\hline
\end{tabular}

The model suggests how these states may be recognized from a set of signs and which actions may be adopted to promote a correct behaviour in each of them. Signs display the following aspects of the subject's mental state:

- value given to the 'correct' behaviour which is the object of the persuasion process;

- $\quad k n o w l e d g e$ of reasons influencing the adoption of a problem behavior;

- belief that (internal and external) conditions exist to change this behavior;

- intention to change own behaviour if wrong; 
- knowledge of an acceptable plan which enables achieving this intention;

- level of perseverance in following the plan.

Actions that may be applied at every state of change respond to the following goals:

- Recognize the situation

- Inform and encourage evaluation processes rather than enforcing persuasion

- Influence intentions

- Check abilities

- Suggest plans

- Support during plan execution.

This model may therefore be adopted as a powerful knowledge source to build cognitive models of the users' attitude and to decide how to tailor advice-giving to their state.

As suggested in (Velicer et al,1998), state of change of behaviour and emotional state are strongly interrelated. For instance, in the precontemplation state subjects may be demoralized about their ability to change, if they tried to do it and failed; in the maintenance state, people are increasingly more confident that they can continue their change; negative affect or emotional distress are indicators of the risk of relapse, and so on. Recognizing some aspects of the emotional state (valence and arousal) may therefore contribute to inferring the state of change and, at the same time, may drive the choice of an appropriate persuasion strategy.

Accurate measurement of the state of change can be performed by asking individuals to respond accurately to a series of unambiguous questions. To this aim, a questionnaire may be administered to the subject at the dialog start. Alternatively, the state of change (and the emotional state) may be inferred progressively during the dialog; uncertainty in knowledge of this data has then to be accepted as a critical feature of dialog simulation, and the dialog modelling method must be adapted to this situation. This is the approach we take in this paper.

\section{Cognitive and Affective Models}

Let us adopt the following notations:

$\mathrm{A}_{\mathrm{i}}, \mathrm{A}_{\mathrm{h}}$ denote the two interlocutors of the dialog; $\mathrm{U}$ denotes, in particular, the user, $\mathrm{S}$ the system; $\mathrm{x}, \mathrm{y}, \mathrm{z}$, denote domain facts ; $\mathrm{g}, \mathrm{g}$ ' denote desired states of the world; e denotes an emotion. The following formulae stay for respective sentences:

$\left(\right.$ Say $\left.\mathrm{A}_{\mathrm{i}} \mathrm{x}\right)$ for "agent $A_{i}$ says $x$ "; $\left(\mathrm{Bel} \mathrm{A}_{\mathrm{i}} \mathrm{x}\right)$ for " $A_{i}$ believes that $x$ "; $\left(\right.$ Goal $\left.\mathrm{A}_{\mathrm{i}} \mathrm{g}\right)$ for " $A_{i}$ wants that g"; $\left(\mathrm{Bel} \mathrm{A}_{\mathrm{i}}\left(\mathrm{Ev}-\mathrm{Ach} \mathrm{A}_{\mathrm{i}} \mathrm{g}\right)\right.$ for " $A_{i}$ believes that its goal $g$ will be achieved sometimes in the future"; (Feels $\mathrm{A}_{\mathrm{i}} \mathrm{e}$ ) for " $A_{i}$ feels the emotion $e^{\text {". }}$

What an agent $A_{i}$ says is a function of its own state of mind and of its goals and beliefs about the interlocutor's state of mind; this communicative act influences $A_{h}$ 's state of mind. According to this model, an agent move (Say $\mathrm{A}_{\mathrm{i}} \mathrm{x}$ ) activates two sorts of processing activities, depending on who $\mathrm{A}_{\mathrm{i}}$ is:

Manipulation: $A_{i}$ is the system and $A_{h}$ is the user. The model allows to forecast the possible effects of a system move on the emotional state of the user and therefore to select the most promising strategy to apply for a given user and in a given situation. 
We represent this model with a dynamic belief network, as described in more detail elsewhere (Carofiglio et al. 2002).

Empathy: we employ a similar model to simulate activation of emotions in the agent, after a user move (see figure 1).

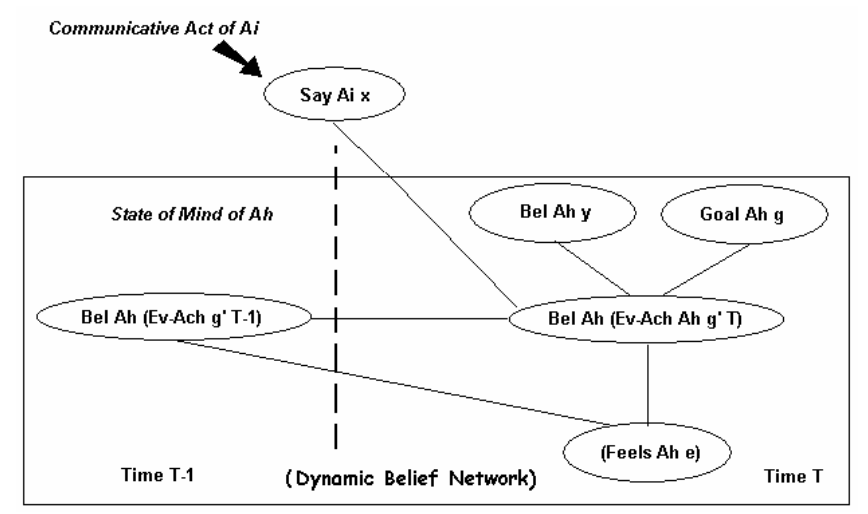

Fig. 1: The emotion activation model

Interpretation: $\mathrm{A}_{\mathrm{i}}$ is the User and $\mathrm{A}_{\mathrm{h}}$ is the system. The model allows to infer the user's state of mind from analysis of her move. We implemented this model with a static belief network whose 'hidden variables' are the state of change and the emotional state and whose 'observables' are the user moves (figure 2).

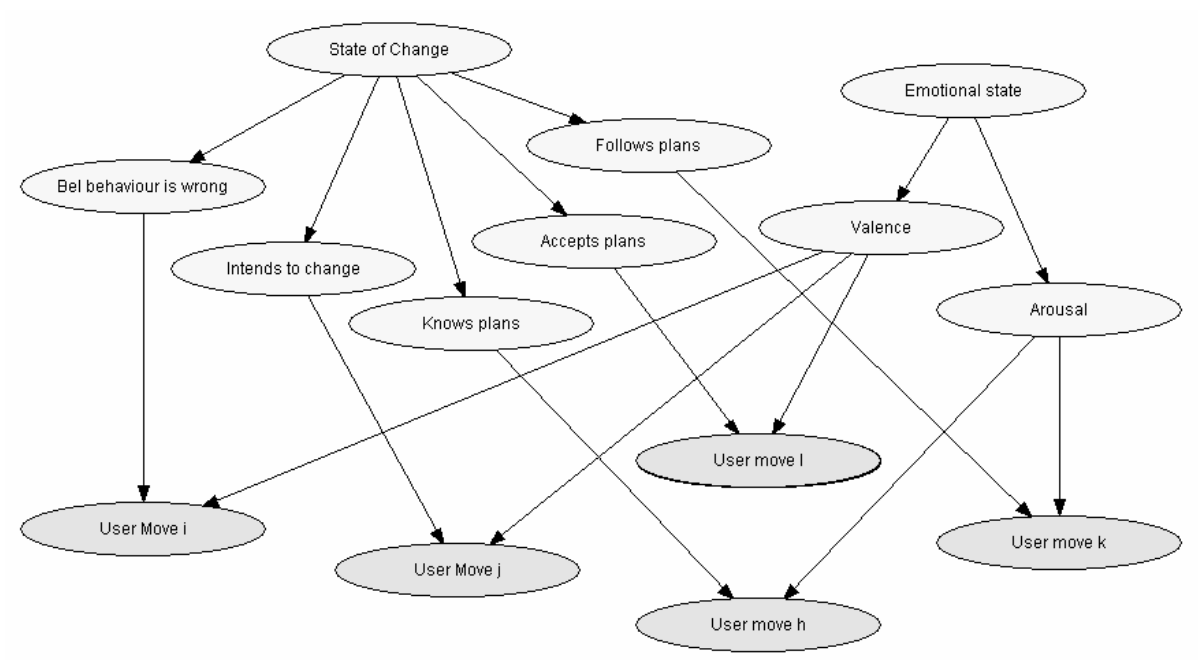

Fig. 2: The User move interpretation model 
As we said in Section 3, the 'state of mind' should be intended, in this case, as a combination of state of change and emotional situation (defined in terms of valence and arousal, as in the circumplex model of affect: Russell, 1980). The intermediate nodes of the network are the aspects of the user's mental state that we described in Section 3. The user may display these cognitive features through one or more communicative acts (leaf nodes). For instance: If the user says "I definitely intend to change my eating habits" or "I want to do every possible effort to get out of this", these moves will be interpreted as a strong intention to change. If he says "I tried several times but I failed", the agent may infer that the user has the intention to change, formulated a plan to achieve this goal but did not go on in applying it. Therefore, reasoning is performed in this BN by introducing evidence in one or more leaf nodes and checking how the probability distribution of the "state of change", the "valence" and the "arousal" variables change.

Application of the three models of manipulation, empathy and interpretation produces a dynamic revision of the mental state of the agent and the user: their beliefs, goals and affective state are updated as far as the dialog proceeds and influence activation of the plans the agent performs to achieve them. We will describe how this is done after shortly reminding the principles behind the information state approach to dialog simulation.

\section{The Information State (IS) Model}

This model was developed in the scope of the TRINDI EC Project (TrindiKit website) as a method to formalize modular dialog simulation systems with a plan-based approach. The information state (IS) is the blackboard in which all data needed to develop the dialog are stored; these data are revised dynamically by means of IS update rules; tasks to perform and plans to achieve them are represented with a logical formalism; select rules establish the next plan and move to perform; a control strategy drives activation of rules; some external modules integrate the functions performed by the dialog manager (Traum and Larsson, 2003).

In simulating our affective dialogs with an IS model, we developed the following components: IS structure, IS updating, Goals and plans and Interaction Manager.

\subsection{IS structure}

The IS is divided, as always, in a 'shared' and a 'private' part. We upgraded its structure with new data, which enable us to represent a BDI\&E model of both the agent and the user (Table 2). The AGENT Model is a subrecord of the SHARED record and includes several fields:

- four stacks (in GOAL), which represent its goals, labelled as 'short-term' or 'long-term', 'achieved' and 'current';

- a set of propositions (in HISTORY) which represent the completed plans; 
- a set of propositions (in IM, 'integrated move'), which represent the moves performed;

- a couple of strings (in EMOTION), which represent the last emotion felt. These data are updated after every user move (section 4).

Table 2

$\begin{array}{ccc}\text { SHARED } & & \text { BEL } \\ & & \text { QUD } \\ & \text { AGENT } & \text { LM } \\ & & \text { GOAL } \\ & & \text { HISTORY } \\ & & \text { IM } \\ & \text { USER } & \text { EMOTION } \\ & & \text { STABLE } \\ \text { PRIVATE } & & \text { UNSTABLE } \\ & \text { PRIVATE } & \text { AGENDA } \\ & & \text { PLAN } \\ & & \text { BEL } \\ & & \text { TMP }\end{array}$

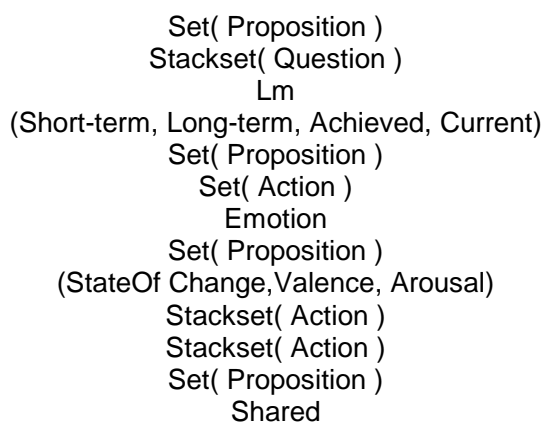

The dialog history is stored in this part of the IS; this information drives applicability of IS update rules. The USER Model is also a subrecord of the SHARED record and includes two sets of propositions:

- permanent characteristics (in the 'STABLE' part) which do not change in the course of the dialog: for instance, 'name', 'age' and 'personality';

- transitory characteristics (in the 'UNSTABLE' part) which are revised during the dialog: for instance, the user's emotional state and her state of change.

\subsection{IS updating}

To select the goals to achieve and the best plans to achieve them, our system needs to employ any information it has got so far about the user. Uncertain default information may be employed to guide the first steps of advice-giving, provided this blurred picture of the user is refined in the course of the dialog, so as to refine also the advice provided. Our simulator therefore needs on one side an uncertain knowledge updating system and, on the other side, a description of the current situation of the two interlocutors and of the dialog history, on which to ground its planning activity.

The logical representation and inference mechanism of TRINDI is unsuited to represent uncertainty in updating the user model and in interpreting her moves. To perform this function, we need some external components which process the static and dynamic belief networks described in Section 4: we implemented these functions by means of Hugin's APIs (HUGIN website).

At the same time, a set of updating rules enables us to import in the IS the results of propagating new evidence in these networks. We defined, in particular, 'empathic' 
rules to update the emotional state of the agent after a user move and 'user updating' rules to revise the 'unstable' part of the user model after interpreting her move.

\subsection{Goals and Plans}

The goals described in Section 3 are not isolated entities: they are related by priority links which establish an order to follow in their consideration according to the following strategies:

- 'recognize the situation' is done until preliminary data have been acquired and the user's state of change may be inferred with a probability above a given threshold;

- 'inform and encourage evaluation' is done in all cases in which the subject does not seem to be really convinced that her behaviour is not correct;

- 'plan suggestion' and 'ability checking' are conditioned to verifying that the subject has got the minimum required level of intention to change her behaviour;

- 'support to plan execution' is conditioned to verifying that the subject did formulate a plan and is following at least its initial steps.

Every goal may be achieved by means of one or more plans, each characterized by a set of applicability conditions on the user state of change and the emotional state of the two interlocutors. A plan Pi includes a main part (the essential of the plan) and a secondary part (with optional details). It may be linked to another plan $\mathrm{Pj}$ by a causal link, to represent that the execution of at least its main part should precede execution of $\mathrm{Pj}$. Introduction of precedence relationships and distinction between main and secondary parts are our solution to the problem of uncertainty in the knowledge of the user state. The system gives the user the opportunity to react to its moves, by fragmenting its plans into short moves and by interleaving suggestions with questions. The user may answer these questions or input new comments; both kinds of moves enable the system to reason on her state and on the emotional effect produced by its move. On the other side, as interpretation of user moves is controversial, dialogue plans are selected by default but may be revised as far as information acquired becomes more clear. When the user model is revised, an on-going plan may be interrupted, to be substituted with a new one. In this case, priority and causal relationships insure that a 'believable' dialog flow is kept also after plans are changed. The distinction between main and secondary parts insures that a goal is achieved with plans at different level of detail, depending on the situation and the dialog history.

\subsection{Interaction Manager}

Effective advice-giving is favoured by a friendly interaction style. In the present prototype, the list of possible user moves in every dialog phase is displayed in a iconic menu, in which the user selects the control which best represents what she would like to say. As an ongoing next step of our research, input will be in free text, with a lin- 
guistic analysis to recognize emotional and state components; this evidence will be propagated in the belief network.

The agent move may be displayed in textual form or may be pronounced by an embodied agent. The agent's body may be selected among a set of options as the most suited to the specific interaction context: GRETA (Pelachaud et al, 1996), MS Agents (MS-Agent website) or Haptek (Haptek website). To this aim, the agent move is transferred to an external body-animation module as an APML string (De Carolis et al et al, 2003) which activates a 'wrapper' to the player (de Rosis et al, 2003). A graphical interface schedules the various functions, activates the related modules and manages input/output.

\section{The Two Examples Commented}

Let us see the dynamics of interpretation and planning in the example dialogs we introduced in Section 3: we show how the system's image of the user and the plans performed evolve in the course of the two dialogs. In both cases, the user is initially thought to be in the 'contemplation' state, with a probability above the threshold. The system then adopts the plan 'Clarify, decision is yours', which suits the goal activated by this state. After move U3, the system feels a light emotion of 'sorry-for', which activates a plan of "empathy display': "I know what you mean". The inferred state of change does not differ from the previous one and the inferred valence is negative. Hence, a plan of 'encourage evaluation of pros and cons of behaviour change' is performed. Comments about the rest of Dialog 1 and about Dialog 2 are similar.

Dialog1: the inferred state does not change

User's state of change: Contemplation ( $\mathrm{p}=25.65)$

S3: I may try to help you, but cannot take a decision in your place.

Plan: Clarify: the decision is yours

U3: I would like to do something but it's so difficult!

Move interpretation: (Say U 'change is difficult')

System emotion: light sorry-for

User's state of change: Contemplation $(p=24.01)$

Valence: negative

S4: I know what you mean: diet is difficult to follow, particularly if you eat out of home because of work. But the effects may be very positive!

Plan: Display empathy; Encourage the evaluation of pros and cons of behaviour change U4: Are you sure?

Move interpretation: (Say U 'doubts pros')

System emotion: sorry-for increases of intensity

User's state of change: Contemplation $(p=25.65)$ 
Valence: negative

S5: Of course! Being in good shape can have beneficial effects on your appearance and health!

Plan: Encourage the evaluation of pros and cons of behaviour change (goes on)

Dialog 2: the inferred state is revised

as in Dialog 1

S4: I know, diet is difficult to follow, particularly if you eat out of home because of work. But the effects may be very positive!

Plan: Encourage the evaluation of pros and cons of behaviour change.

U4: I know! I definitely intend to change my habits.

Move interpretation: (Say $U$ 'intends to change')

System emotion: light hope

User's state of change: Preparation $(p=24.35)$

Valence: positive

S5: Good! Why, for instance, don't you try to avoid daily lunches in a bar or high-fat sandwiches?

Plan: Display satisfaction; Encourage initial steps

U5: Everyday you mean? I may try, but must find an alternative.

Move interpretation: (Say U 'will try' \& Ask U 'how')

System emotion: hope increases of intensity

User's state of change: Preparation $(p=31.12)$

Valence: positive

S6: You may bring something from home: some fresh fruits or vegetables, for instances. And have a cup of tea or a fruit juice.

Plan: Encourage initial steps (goes on)

\section{Final Remarks and Related Work}

In this paper, we advocate that, to simulate affective dialogs, recognition of the emotional state, the mood and the attitude of the user should be integrated with an interpretation of these features, which helps in understanding what produced this affective state and how the following course of the dialog should be adapted to this situation. As we said, so far we do not deal with the recognition problem (which, as it is well known, may be based on analysis of visual, prosodic and linguistic data) but only with their interpretation. Being aware that this interpretation is uncertain, we propose to adopt one of the typical models of uncertain reasoning: static and dynamic belief models (Ball, 2003). We demonstrate how we integrated these models in an information-state approach to dialog modelling. The reason why we prefer this approach to state transition models is that affective advice-giving dialogs are highly dynamic: 
inference about the affective state of the user may evolve during the dialog, and the system has to revise its behaviour to adapt to the situation. To this aim, it has not only to 'recognize' that the user is (say) 'demoralized' or 'confident' about changing her behaviour, but also to infer which is the reason of this affective state: this will enable it to focus its repair strategy on the likely causes of the recognized situation.

The body of experience to which our work is more closely related is Cassell and Bickmore's research on 'relational agents' (2003) and, more specifically, LAURA (Bickmore, 2003). Although LAURA deals with advice about physical exercise rather than healthy eating, the background of our dialog simulators is the same as LAURA's: in particular, Prochaska and colleagues' state of change model. There are, however, several differences between our approaches and experiences. LAURA is aimed at providing long-term advice and follow-up: so, dialogs are repeated regularly to evaluate the effect of advice provided in the previous phases and to assess whether and how the state of change of the subject evolved as expected. In every state, though, limited space is left to handling of uncertainty, no user model is built and the system feedback is not tailored to the emotional state of the user. Consequently, the interactions are represented as state-transition models and follow a predefined template. While this approach is powerful and efficient in the situation envisaged in LAURA, it is not so when information states are not finite in principle or are so many, that the complexity of the ATNs would become very high. It is not convenient also when the motivations for selecting the next dialogue move relies only on part of the information available, rather than on the whole state. This occurs when the goal is to adapt the dialog to the user's state and also to the agent's emotional reaction.

As we said, so far we recognize the state of the user from a predefined list of inputs: in an ongoing research, we are studying how to infer it from linguistic analysis of free text. Other authors applied this method to infer 'personality traits' from analysis of emails (Gill and Oberlander, 2002) or combined prosodic and linguistic information to recognize the emotional state of the interlocutor (Lee at al 2002) and the accuracy of recognition was rather high, if compared with the simplicity of the acquisition method. Parameters in our BN-based cognitive models have been settled after a purely subjective evaluation. However, state of change models are applied to innumerable cases of health advice, and local distributions of people across the various stages may be obtained from health services: we therefore plan to refine calibration of our models after analyzing experimental data from this kind of sources.

\section{Acknowledgements}

We thank Berardina Nadja De Carolis for helpful suggestions on the reactive planning aspects of this research and Giuseppe Cellamare for cooperating to implementation of the prototype. 


\section{References}

1. Ball, E. A bayesian heart: Computer recognition and simulation of emotion. In R.Trappl, P.Petta and S. Payr (Eds): Emotions in Humans and Artifacts. The MIT Press, 2003.

2. Bickmore T. Relational Agents: Effecting change through human-computer relationships. PhD Thesis. 2003

3. Carofiglio, V., de Rosis, F., Grassano, R. (ed.) Dynamic models of mixed emotion activation. In D.Canamero and R.Aylett: Animating expressive characters for social interaction. John Benjamins 2003.

4. Cassell, J. and Bickmore, T. Negotiated collusion: Modeling social language and its relationship effects in intelligent agents. Usr Modeling and User-Adapted Interaction. 13, 89-132, 2003.

5. De Carolis, B., Bilvi, M. and Pelachaud,C. APML, a mark-up language for believable behavior generation. In H Prendinger and M Ishizuka (Eds): "Life-like Characters. Tools, Affective Functions and Applications". Springer, 2003.

6. de Rosis, F. Towards merging cognition and affect in HCI. Applied Artificial Intelligence (2002).

7. de Rosis, F., Pelachaud, C., Poggi, I., Carofiglio, V., De Carolis, B. (ed.) From Greta's Mind to her Face: Modeling the Dynamics of Affective States in a Conversational Embodied Agent. International Journal of Human-Computer Studies. B.R.Gaines. Vol.59 (1-2) (2003).

8. Gill, A..J., Oberlander, J. Taking care of the linguistic features of extraversion. In Proceedings of the 24th Annual Conference of the Cognitive Science Society. Fairfax VA, August (2002) 363-368.

9. Lee, C.M., Narayanan, S.S., Pieraccini, R. Combining acoustic and language information for emotion recognition. Proceedings of ICSLP (2002).

10. Haptek website: www.haptek.com

11. HUGIN website: http://www.hugin.com/

12. MS-Agent: http://www.microsoft.com/msagent/

13. Ortony A., Clore G.L., Collins A. The cognitive structure of emotions. Cambridge University Press (1988).

14. Pelachaud, C., Badler, N.I., Steedman, M. Generating Facial Expressions for Speech. Cognitive Science, 20,1 (1996).

15. Petty, R.E., Cacioppo, J.T. The Elaboration likelihood model of persuasion. Advances in Experimental Social Psychology, 19 (1986).

16. Prochaska, J., Di Clemente, C., Norcross, H. In search of how people change: applications to addictive behavior. Americal Psychologist, 47 (1992) 1102-1114

17. Russell J.A.(1980). A Circumplex model of Affect. Journal of Personality and Social Psychology, 39, 6.

18. Traum, D.R., Larsson, S. (ed.) The Information State approach to dialogue management. 'Current and New Directions in Discourse and Dialogue', Jan van Kuppevelt and Ronnie Smith, Kluwer. (2003)

19. TRINDIKIT 2.0 Manual: http://www.ling.gu.se/research/projects/trindi

20. Velicer, W.F, Prochaska, J.O, Fava, J.L., Norman, G.J, Redding, C.A . Smoking cessation and stress management: Applications of the Transtheoretical Model of behaviour change. Homeostasis, 38, 216-233. (1998) 